$\xi=\square$

\title{
A variety of exact analytical solutions of extended shallow water wave equations via improved $\left(G^{\prime} / G\right)$-expansion method
}

\author{
Faisal Hawlader *, Dipankar Kumar \\ Department of Mathematics, Bangabandhu Sheikh Mujibur Rahman Science and Technology University, Gopalganj-8100, Bangladesh \\ *Corresponding author E-mail: faisalhawlader260@gmail.com
}

\begin{abstract}
In this present work, we have established exact solutions for $(2+1)$ and $(3+1)$ dimensional extended shallow-water wave equations involving parameters by applying the improved $\left(\mathrm{G}^{\prime} / \mathrm{G}\right)$-expansion method. Abundant traveling wave solutions with arbitrary parameter are successfully obtained by this method, and these wave solutions are expressed in terms of hyperbolic, trigonometric, and rational functions. The improved $\left(\mathrm{G}^{\prime} / \mathrm{G}\right)$-expansion method is simple and powerful mathematical technique for constructing traveling wave, solitary wave, and periodic wave solutions of the nonlinear evaluation equations which arise from application in engineering and any other applied sciences. We also present the 3D graphical description of the obtained solutions for different cases with the aid of MAPLE 17.
\end{abstract}

Keywords: Extended Shallow Water Wave Equations; Exact Solutions; Improved $\left(G^{\prime} / G\right)$-Expansion Method; Nonlinear Partial Differential Equations.

\section{Introduction}

A great deal of work has been carried out in order to determine exact traveling wave solutions for nonlinear evaluation equations (NLEEs) in all areas of Science and Engineering. In the nonlinear sciences, it is well known that many NLEEs are widely used as to describe the complex physical phenomena, such as Plasma physics, Chemical physics, Solid-state physics, Chemical kinematics, Biology, Optical fibers, Fluid mechanics, etc., It is well known that searching for an exact solution of nonlinear evolution equation arising in mathematical physics plays an important role in the study of nonlinear physical phenomena. In the recent past, many researchers have implemented various analytical methods [1-24] to seek traveling wave solutions of different nonlinear partial differential equations. Many powerful methods have been successfully developed by diverse groups of mathematicians and physicists, such as, Hirota's bilinear method [1], the tanh-function method [2], [3], the extended than-function method [4], the exp -function method [5-7], sine-cosine method [8], the Inverse scattering transforms [9], the Jacobi elliptic function expansion [10], [11], the homogeneous balance method [12], the Homotopy perturbation methods [13], auxiliary equation method [14], the first integral method [15-17], the tanh-coth method [18], the Cole-Holf transformation method [19], the $\left(G^{\prime} / G\right)$-expansion method [20-22], the improved $(G \% G)$-expansion method [23], the Enhance $\left(G^{\prime} / G\right)$-expansion method [24] and so on. The improved $\left(G^{\prime} / G\right)$-expansion method [23] is a direct and effective algebraic method for handling nonlinear evaluations equations, and the author [23] have been applied the improved $(G \% G)$-expansion method for solving modified Korteweg-de Vries equation.

The objective of this study is to apply the improved $\left(G^{\prime} / G\right)$ expansion method to find the exact traveling wave solutions of the $(2+1)$ and $(3+1)$ dimensional extended shallow-water wave equations involving parameters. The extended model of shallowwater waves equations important partial differential equations of nonlinear dispersive waves. The $(2+1)$ dimensional shallow-water wave equations [7], [22-23].

$$
\begin{aligned}
& u_{x t}+u_{x x y}-2 u_{x x} u_{y}-4 u_{x} u_{x y}=0 \\
& u_{y t}+u_{x x y}-3 u_{x x} u_{y}-3 u_{x} u_{x y}=0
\end{aligned}
$$

Were studied. Both equations reduce to potential KdV equation for $y=x$.The difference between the two models (1) and (2) is that $x$ replaces $y$ in the term $u_{x t}$ and in the coefficients of the other terms. The $(3+1)$ dimensional shallow water wave equations [7], [22-23]

$$
\begin{aligned}
& u_{x x t}+u_{x x y z}-2\left(u_{x x} u_{y z}+u_{y} u_{x x z}\right)-\left(4 u_{x} u_{x y z}+u_{x z} u_{x y}\right)=0 \\
& u_{y z}+u_{x x y z}-6 u_{x} u_{x y z}-6 u_{x z} u_{x y}=0
\end{aligned}
$$

Were studied. Both equations reduce to potential KdV equation for $y=x$.The difference between the two models (3) and 4) is that $x$ replaces $y$ in the term $u_{x x t}$.

In this present work, we will solve four extended $(2+1)$ and $(3+1)$ dimensional shallow water wave equations in that were solved by authors [7], [18-19], [22].

$$
\begin{aligned}
& u_{y t}+u_{x x y}-3 u_{x x} u_{y}-3 u_{x} u_{x y}+\alpha u_{x y}=0 \\
& u_{x t}+u_{x x y y}-2 u_{x x} u_{y}-4 u_{x} u_{x y}+\alpha a u_{x y}=0 \\
& u_{y x t}+u_{x x y z}-6 u_{x} u_{x y z}-6 u_{x z} u_{x y}+\alpha u_{x y z}=0
\end{aligned}
$$




$$
u_{x z t}+u_{x x y z}-2\left(u_{x x} u_{y z}+u_{y} u_{x x z}\right)-4\left(u_{x} u_{x y z}+u_{x z} u_{x y}\right)+\alpha u_{x y z}=0
$$

These shallow-water wave equations play a major role in the study of nonlinear dispersive waves. The general idea of dispersive waves oriented from the problem of water waves. The shallowwater wave equations describe the evaluation of incompressible flow, neglecting density change along the depth; shallow-water wave equations are applicable to cases where the horizontal scale of the flow is much bigger than the depth of fluid [21].

The article is arranged as follows: In section 2, the methodology is discussed. In section 3, we apply this method to solve the nonlinear evaluation equations point out above. We will discuss the solution of the result and its discussion in section 4 . In section 5 , we present the graphical representation, and finally we conclude this article in section 6.

\section{Methodology}

Suppose the general nonlinear partial differential equation in three independent variables $x$ and $t$ is given by

$N\left(u, u_{t}, u_{x}, u_{y}, u_{x y}, u_{x x}, u_{x t}, u_{y t}, u_{t t} \ldots\right)=0$

Where $u=u(x, y, t)$ is an unknown function, $N$ is a polynomial in $u=u(x, y, t)$ and the subscripts signify the partial derivatives and the nonlinear term are involved.

The main steps of the improved $\left(G^{\prime} / G\right)$-expansion method as follows:

Step 1: Consider the traveling wave variable

$u(x, y, t)=u(\xi), \xi=x+y-c t$

Where $c$ is the speed of the traveling wave.

Now using equation (10), the equation (9) is transformed into an ordinary differential equation for $u=u(\xi)$ in the form

$P\left(u, u^{\prime}, u^{\prime \prime}, u^{\prime \prime}, \ldots\right)=0$

Where $P$ is a polynomial in $u(\xi)$ and its total derivatives. The superscripts stand for the ordinary derivatives with respect to $\xi$.

Step 2: Suppose that the traveling waves solution of equation (12) can be expressed by a polynomial in $F(\xi)$ as the following form:

$u(\xi)=\sum_{i=0}^{n} \alpha_{i}(m+F(\xi))^{i}+\sum_{i=1}^{n} \beta_{i}(m+F(\xi))^{-i}$

Where $F(\xi)=\frac{G^{\prime}(\xi)}{G(\xi)}, \alpha_{n}$ and $\beta_{n}$ are not zero simultaneously.

Also, the function $G(\xi)$ is the solution of the linear ordinary differential equation

$G^{\prime \prime}(\xi)+\lambda G^{\prime}(\xi)+\mu G(\xi)=0$

Where $\mathrm{G}^{\prime \prime}=\frac{d^{2} G}{d \xi^{2}}, G^{\prime}=\frac{d G}{d \xi}$ and $\lambda, \mu$ are real constants to be determined later, $n$ is a positive integer to be determined later and $n$ can be determined by considering the homogeneous balance between the highest order derivatives and the highest order nonlinear terms appearing in ODE equation (11).

The solutions of equation (13) can be written as follows:

\subsection{Hyperbolic function solution: (when $\Omega=\lambda^{2}-4 \mu>0$ )}

$F_{1}=\frac{\sqrt{\Omega}}{2} \operatorname{coth}\left(A+\frac{\sqrt{\Omega}}{2} \xi\right)-\frac{\lambda}{2}$

$F_{2}=\frac{\sqrt{\Omega}}{2} \tanh \left(A+\frac{\sqrt{\Omega}}{2} \xi\right)-\frac{\lambda}{2}$

\subsection{Trigonometric function solution: (when $\Omega=\lambda^{2}-4 \mu<0$ )}

$$
\begin{aligned}
& F_{3}=\frac{\sqrt{-\Omega}}{2} \cot \left(A+\frac{\sqrt{-\Omega}}{2} \xi\right)-\frac{\lambda}{2} \\
& F_{4}=\frac{\sqrt{-\Omega}}{2} \tan \left(A-\frac{\sqrt{-\Omega}}{2} \xi\right)-\frac{\lambda}{2}
\end{aligned}
$$

\subsection{Rational function solution: (when $\Omega=\lambda^{2}-4 \mu=0$ )}

$$
F_{5}=\frac{B}{A+B \xi}-\frac{\lambda}{2}
$$

Step 3: Substituting equation (12) into (11) and using (13), with the value of $n$ obtained in step 2, then we obtain polynomials in $F(\xi)^{i}$ and $F(\xi)^{-i} \quad(i=0,1,2,3, \ldots)$, separate all terms with same order of $F(\xi)^{i}$ and $F(\xi)^{-i}$, then equating each coefficient of the polynomials to zero, yield a system of algebraic equations for $\alpha_{n}$, $\beta_{n}, c, \lambda$ and $\mu$

Step 4: Solve the system of algebraic equations which are found in step - 3 with the aid of algebraic software Maple to obtain values for $\alpha_{n}, \beta_{n}, m$ and $c$.

Step 5: Substitute obtained values in step - 4, in equation (12) along with equation (13) with the value of $n$, we obtain some exact traveling wave solutions of the nonlinear equation (9).

\section{Application of the method}

In this section, we implement the method described in section 2 to find the exact analytical solutions of $(2+1)$ dimensional first and second extended shallow water wave equations via improved $\left(G^{\prime} / G\right)$-expansion method.

\subsection{The first extended shallow water wave equation}

In this present work, we consider the extended shallow water wave equation in $(2+1)$ dimensions of the following form [7], [22]

$u_{x t}+u_{x x x y}-2 u_{x x} u_{y}-4 u_{x} u_{x y}+\beta u_{x y}=0$

Where $\beta$ is a non-zero constant. According to the method by using the traveling wave variable

$u(x, t)=u(\xi), \xi=k x+r y-c t$,

Where $c$ is the speed of the traveling wave, transform equation (14) into an ordinary differential equation, integrating ODE and neglecting constants of integration, we have

$k^{2} r u^{\prime \prime}+(r \beta-c) u^{\prime}-3 k r\left(u^{\prime}\right)^{2}=0$

Where the prime denotes differential with respect to $\xi$. Balancing $u$ "' with $\left(u^{\prime}\right)^{2}$, we get $n+3=2(n+1)$ Hence $n=1$

Hence for $n=1$ Eq. (12) reduces to 
$u(\xi)=\alpha_{0}+\alpha_{1}(m+F)+\beta_{1}(m+F)^{-1}$

Now substituting Eq. (17) along with Eq. (13) into Eq. (16), we get a polynomial in $F(\xi)$.Equating the coefficient of same power of $F(\xi)$, we attain the following system of algebraic equations:

$6 k^{2} r \alpha_{1}+3 k r \alpha_{1}^{2}=0$

$24 k^{2} m r \alpha_{1}+12 k^{2} r \lambda \alpha_{1}+12 k m r \alpha_{1}^{2}+6 k r \lambda \alpha_{1}^{2}=0$

$36 k^{2} m^{2} r \alpha_{1}+48 k^{2} m r \lambda \alpha_{1}+7 k^{2} r \lambda^{2} \alpha_{1}+18 k m^{2} r \alpha_{1}^{2}+24 k m r \lambda \alpha_{1}^{2}$

$+3 k r \lambda^{2} \alpha_{1}^{2}+8 k^{2} r \mu \alpha_{1}+6 k \mu r \alpha_{1}^{2}-6 k r \alpha_{1} \beta_{1}+r \beta \alpha_{1}-c \alpha_{1}=0$

$24 k^{2} m^{3} r \alpha_{1}+72 k^{2} m^{2} r \lambda \alpha_{1}+28 k^{2} m r \lambda^{2} \alpha_{1}+k^{2} r \lambda^{3} \alpha_{1}+12 k m^{3} r \alpha_{1}^{2}$

$+36 k m^{2} r \lambda \alpha_{1}^{2}+12 k m r \lambda^{2} \alpha_{1}^{2}+32 k^{2} m \mu r \alpha_{1}+8 k^{2} \mu r \lambda \alpha_{1}$

$+24 k m \mu r \alpha_{1}^{2}+6 k \mu r \lambda \alpha_{1}^{2}-12 k m r \alpha_{1} \beta_{1}-12 k r \lambda \alpha_{1} \beta_{1}$

$+4 m r \beta \alpha_{1}+r \beta \lambda a_{1}-4 c m \alpha_{1}-c \lambda \alpha_{1}=0$

$3 k r m^{4} \alpha_{1}^{2}+3 k r \mu^{2} \alpha_{1}^{2}+6 k^{2} r m^{4} \alpha_{1}-6 k^{2} r m^{2} \beta_{1}+2 k^{2} r \mu^{2} \alpha_{1}$

$-k^{2} r \lambda^{2} \beta_{1}-2 k^{2} r \mu \beta_{1}+6 m^{2} r \beta \alpha_{1}-4 c m \lambda \alpha_{1}+\mu r \beta \alpha_{1}-6 c m^{2} \alpha_{1}$

$-c \mu \alpha_{1}-r \beta \beta_{1}+3 k r \beta_{1}^{2}+c \beta_{1}+32 k^{2} r m \mu \lambda \alpha_{1}+24 k r m \mu \lambda \alpha_{1}^{2}$

$-24 k r m \lambda \alpha_{1} \beta_{1}-12 k r \mu \alpha_{1} \beta_{1}+48 k^{2} r m^{3} \lambda \alpha_{1}+42 k^{2} r m^{2} \lambda^{2} \alpha_{1}$

$+4 k^{2} r m \lambda^{3} \alpha_{1}+48 k^{2} r m^{2} \mu \alpha_{1}+k^{2} r \mu \lambda^{2} \alpha_{1}+6 k^{2} r m \lambda \beta_{1}$

$+4 \mathrm{rm} \beta \lambda \alpha_{1}+24 \mathrm{krm}^{3} \lambda \alpha_{1}^{2}+18 \mathrm{krm}^{2} \lambda^{2} \alpha_{1}^{2}+36 \mathrm{krm}^{2} \mu \alpha_{1}^{2}$

$-6 k r m^{2} \alpha_{1} \beta_{1}-6 k r \lambda^{2} \alpha_{1} \beta_{1}=0$

$6 k r \lambda \beta_{1}^{2}-k^{2} r \lambda^{3} \beta_{1}+4 m^{3} r \beta \alpha_{1}-6 c m^{2} \lambda \alpha_{1}-4 c m \mu \alpha_{1}-2 m r \beta \beta_{1}$ $-r \beta \lambda \beta_{1}-4 c m^{3} \alpha_{1}+2 c m \beta_{1}+c \lambda \beta_{1}-24 k r m \mu \alpha_{1} \beta_{1}-12 k r \mu \lambda \alpha_{1} \beta_{1}$ $+48 k^{2} \mathrm{rm}^{2} \mu \lambda \alpha_{1}+4 k^{2} r m \mu \lambda^{2} \alpha_{1}+36 \mathrm{krm}^{2} \mu \lambda \alpha_{1}^{2}-12 \mathrm{krm}^{2} \lambda \alpha_{1} \beta_{1}$

$-12 k r m \lambda^{2} \alpha_{1} \beta_{1}+12 k^{2} r m^{4} \lambda \alpha_{1}+28 k^{2} r m^{3} \lambda^{2} \alpha_{1}+6 k^{2} r m^{2} \lambda^{3} \alpha_{1}$

$+32 k^{2} r m^{3} \mu \alpha_{1}-12 k^{2} r m^{2} \lambda \beta_{1} 8 k^{2} r m \mu^{2} \alpha_{1}+10 k^{2} r m \lambda^{2} \beta_{1}$

$+8 k^{2} r m \mu \beta_{1}-8 k^{2} r \mu \lambda \beta_{1}+6 m^{2} r \beta \lambda \alpha_{1}+4 m \mu r \beta \alpha_{1}+6 k r m^{4} \lambda \alpha_{1}^{2}$

$+12 \mathrm{krm}^{3} \lambda^{2} \alpha_{1}^{2}+24 \mathrm{krm}^{3} \mu \alpha_{1}^{2}+12 \mathrm{krm} \mu^{2} \alpha_{1}^{2}=0$

$3 k r \lambda^{2} \beta_{1}^{2}+6 k r \mu \beta_{1}^{2}-8 k^{2} r \mu^{2} \beta_{1}+m^{4} r \beta \alpha_{1}-4 c m^{3} \lambda \alpha_{1}-6 c m^{2} \mu \alpha_{1}$

$-m^{2} r \beta \beta_{1}+2 c m \lambda \beta_{1}-\mu r \beta \beta_{1}-24 k r m \mu \lambda \alpha_{1} \beta_{1}-c m^{4} \alpha_{1}+c^{2} \beta_{1}$

$+c \mu \beta_{1}-12 k m^{2} \mu \alpha_{1} \beta_{1}+32 k^{2} r m^{3} \mu \lambda \alpha_{1}+6 k^{2} r m^{2} \mu \lambda^{2} \alpha_{1}$

$+20 k^{2} r m \mu \lambda \beta_{1}+24 k r m^{3} \mu \lambda \alpha_{1}^{2}-6 k m^{2} \lambda^{2} \alpha_{1} \beta_{1}-6 k r \mu^{2} \alpha_{1} \beta_{1}$

$+7 k^{2} r m^{4} \lambda^{2} \alpha_{1}+4 k^{2} r m^{3} \lambda^{3} \alpha_{1}+8 k^{2} r m^{4} \mu \alpha_{1}+12 k^{2} r m^{2} \mu^{2} \alpha_{1}$

$-7 k^{2} r m^{2} \lambda^{2} \beta_{1}+4 k^{2} r m \lambda^{3} \beta_{1}-8 k^{2} r m^{2} \mu \beta_{1}-7 k^{2} r \mu \lambda^{2} \beta_{1}+4 m^{3} r \beta \lambda \alpha_{1}$

$+6 m^{2} \mu r \beta \alpha_{1}-2 m r \beta \lambda \beta_{1}+3 k r m^{4} \lambda^{2} \alpha_{1}^{2}+6 k r m{ }^{4} \mu+\alpha_{1}^{2}$

$+18 \mathrm{krm}^{2} \mu^{2} \alpha_{1}^{2}=0$

$8 k^{2} r m^{4} \mu \lambda \alpha_{1}+12 k r m^{3} \mu^{2} \alpha_{1}^{2}+6 k r \mu \lambda \beta_{1}^{2}-k^{2} r m^{2} \lambda^{3} \beta_{1}$

$+k^{2} r m^{4} \lambda^{3} \alpha_{1}+8 k^{2} r m \mu^{2} \beta_{1}-c m^{4} \lambda \alpha_{1}+10 k^{2} r m \mu \lambda^{2} \beta_{1}$

$+4 m^{3} \mu r \beta \alpha_{1}-4 \mathrm{~cm}^{3} \mu \alpha_{1}-12 k^{2} r \mu^{2} \lambda \beta_{1}+6 \mathrm{krm}^{4} \mu \lambda \alpha_{1}^{2}-$

$12 k r m \mu^{2} \alpha_{1} \beta_{1}-m^{2} r \beta \lambda \beta_{1}+2 c m \mu \beta_{1}+8 k^{2} r^{3} \mu^{2} \alpha_{1}-$

$8 k^{2} r m^{2} \mu \lambda \beta_{1}-12 k r m^{2} \mu \lambda \alpha_{1} \beta_{1}+c m^{2} \lambda \beta_{1}-2 m \mu r \beta \beta_{1}$

$+m^{4} r \beta \lambda \alpha_{1}+4 k^{2} r m^{3} \mu \lambda^{2} \alpha_{1}=0$

$-6 k r m^{2} \mu^{2} \alpha_{1} \beta_{1}-6 k^{2} r \mu^{3} \beta_{1}+c m^{2} \mu \beta_{1}+k^{2} r m^{4} \mu \lambda^{2} \alpha_{1}$

$+3 k r m^{4} \mu^{2} \alpha_{1}^{2}+3 k r \mu^{2} \beta_{1}^{2}+2 k^{2} r m^{4} \mu^{2} \alpha_{1}+$

$6 k^{2} r m \mu^{2} \lambda \beta_{1}-k^{2} r m^{2} \mu \lambda^{2} \beta_{1}-2 k^{2} r m^{2} \mu^{2} \beta_{1}-$

$c m^{4} \mu \alpha_{1}+m^{4} \mu r \beta \alpha_{1}-m^{2} \mu r \beta \beta_{1}=0$

Solving the above system of equations for $\alpha_{0}, \alpha_{1}, \beta_{1}, m$ and $c$ with MAPLE 17, we get the following values:

Set 1:

$$
\begin{aligned}
& c=k^{2} r \lambda^{2}-4 k^{2} \mu r+r \beta, m=m, \alpha_{0}=\alpha_{0} \\
& \alpha_{1}=0, \beta_{1}=2 k m^{2}-2 k m \lambda+2 k \mu
\end{aligned}
$$

Set 2:

$c=4 k^{2} r \lambda^{2}-16 k^{2} \mu r+r \beta, m=\frac{1}{2} \lambda, \alpha_{0}=\alpha_{0}$,

$\alpha_{1}=-2 k, \beta_{1}=-\frac{1}{2} k \lambda^{2}+2 k \mu$

Set 3:

$$
\begin{aligned}
& c=4 k^{2} r \lambda^{2}-16 k^{2} \mu r+r \beta, m=\frac{1}{2} \lambda, \alpha_{0}=\alpha_{0} \\
& \alpha_{1}=-2 k, \beta_{1}=-\frac{1}{2} k \lambda^{2}+2 k \mu
\end{aligned}
$$

\subsubsection{Hyperbolic function solutions}

When, $\Omega=\lambda^{2}-4 \mu>0$ we obtain the hyperbolic function solutions of Eq. (14)

Family 1:

$$
\begin{aligned}
& u_{1}(\xi)=\alpha_{0}-2 k\left(m+\frac{1}{2} \sqrt{\Omega} \operatorname{coth}\left(A+\frac{1}{2} \sqrt{\Omega} \xi\right)-\frac{1}{2} \lambda\right) \\
& u_{2}(\xi)=\alpha_{0}-2 k\left(m+\frac{1}{2} \sqrt{\Omega} \tanh \left(A+\frac{1}{2} \sqrt{\Omega} \xi\right)-\frac{1}{2} \lambda\right)
\end{aligned}
$$

Where $\xi=k x+r y-\left(k^{2} r \lambda^{2}-4 k^{2} \mu r+r \beta\right) t$

Family 2:

$$
\begin{aligned}
& u_{3}(\xi)=\alpha_{0}+\frac{2 k m^{2}-2 k m \lambda+2 k \mu}{m+\frac{1}{2} \sqrt{\Omega} \operatorname{coth}\left(A+\frac{1}{2} \sqrt{\Omega} \xi\right)-\frac{1}{2} \lambda} \\
& u_{4}(\xi)=\alpha_{0}+\frac{2 k m^{2}-2 k m \lambda+2 k \mu}{m+\frac{1}{2} \sqrt{\Omega} \tanh \left(A+\frac{1}{2} \sqrt{\Omega} \xi\right)-\frac{1}{2} \lambda}
\end{aligned}
$$

Where $\xi=k x+r y-\left(k^{2} r \lambda^{2}-4 k^{2} \mu r+r \beta\right) t$

Family 3:

$$
\begin{array}{r}
u_{5}(\xi)=\alpha_{0}-k \sqrt{\Omega} \operatorname{coth}\left(A+\frac{1}{2} \sqrt{\Omega} \xi\right) \\
+\frac{2\left(-\frac{1}{2} k \lambda^{2}+2 k \mu\right)}{\sqrt{\Omega} \operatorname{coth}\left(A+\frac{1}{2} \sqrt{\Omega} \xi\right)} \\
u_{6}(\xi)=\alpha_{0}-k \sqrt{\Omega} \tanh \left(A+\frac{1}{2} \sqrt{\Omega} \xi\right) \\
+\frac{2\left(-\frac{1}{2} k \lambda^{2}+2 k \mu\right)}{\sqrt{\Omega} \tanh \left(A+\frac{1}{2} \sqrt{\Omega} \xi\right)}
\end{array}
$$

Where $\xi=k x+r y-\left(4 k^{2} r \lambda^{2}-16 k^{2} \mu r+r \beta\right) t$

\subsubsection{Trigonometric function solutions}

When $\Omega=\lambda^{2}-4 \mu<0$, we obtain the trigonometric function solutions of Eq. (14)

Family 4:

$u_{7}(\xi)=\alpha_{0}-2 k\left(m+\frac{1}{2} \sqrt{-\Omega} \cot \left(A+\frac{1}{2} \sqrt{-\Omega} \xi\right)-\frac{1}{2} \lambda\right)$ 
$u_{8}(\xi)=\alpha_{0}-2 k\left(m+\frac{1}{2} \sqrt{-\Omega} \tan \left(A-\frac{1}{2} \sqrt{-\Omega} \xi\right)-\frac{1}{2} \lambda\right)$

Where $\xi=k x+r y-\left(k^{2} r \lambda^{2}-4 k^{2} \mu r+r \beta\right) t$

Family 5:

$$
\begin{aligned}
& u_{9}(\xi)=\alpha_{0}+\frac{2 k m^{2}-2 k m \lambda+2 k \mu}{m+\frac{1}{2} \sqrt{-\Omega} \cot \left(A+\frac{1}{2} \sqrt{-\Omega} \xi\right)-\frac{1}{2} \lambda} \\
& u_{10}(\xi)=\alpha_{0}+\frac{2 k m^{2}-2 k m \lambda+2 k \mu}{m+\frac{1}{2} \sqrt{-\Omega} \tan \left(A-\frac{1}{2} \sqrt{-\Omega} \xi\right)-\frac{1}{2} \lambda}
\end{aligned}
$$

Where $\xi=k x+r y-\left(k^{2} r \lambda^{2}-4 k^{2} \mu r+r \beta\right) t$

Family 6:

$$
\begin{array}{r}
u_{11}(\xi)=\alpha_{0}-k \sqrt{-\Omega} \cot \left(A+\frac{1}{2} \sqrt{-\Omega} \xi\right) \\
+\frac{2\left(-\frac{1}{2} k \lambda^{2}+2 k \mu\right)}{\sqrt{-\Omega} \cot \left(A+\frac{1}{2} \sqrt{-\Omega} \xi\right)} \\
u_{12}(\xi)=\alpha_{0}-k \sqrt{-\Omega} \tan \left(A-\frac{1}{2} \sqrt{-\Omega \mu} \xi\right) \\
+\frac{2\left(-\frac{1}{2} k \lambda^{2}+2 k \mu\right)}{\sqrt{-\Omega} \tan \left(A-\frac{1}{2} \sqrt{-\Omega} \xi\right)}
\end{array}
$$

Where $\xi=k x+r y-\left(4 k^{2} r \lambda^{2}-16 k^{2} \mu r+r \beta\right) t$

\subsubsection{Rational function solutions}

When $\lambda^{2}-4 \mu=0$, we obtain the following solutions of Eq. (14). Family 7:

$u_{13}(\xi)=\alpha_{0}-2 k\left(m+\frac{B}{B \xi+A}-\frac{1}{2} \lambda\right)$

Where $\xi=k x+r y-\left(k^{2} r \lambda^{2}-4 k^{2} \mu r+r \beta\right) t$

Family 8:

$$
u_{14}(\xi)=\alpha_{0}+\frac{2 k m^{2}-2 k m \lambda+2 k \mu}{m+\frac{B}{B \xi+A}-\frac{1}{2} \lambda}
$$

Where $\xi=k x+r y-\left(k^{2} r \lambda^{2}-4 k^{2} \mu r+r \beta\right) t$

Family 9:

$u_{15}(\xi)=\alpha_{0}-\frac{2 k B}{B \xi+A}+\frac{\left(-\frac{1}{2} k \lambda^{2}+2 k \mu\right)(B \xi+A)}{B}$

Where $\xi=k x+r y-\left(4 k^{2} r \lambda^{2}-16 k^{2} \mu r+r \beta\right) t$

\subsection{The second extended shallow water wave equation}

In this section, we consider the extended shallow water wave equation in $(2+1)$ dimensions of the following form [7], [22]. $u_{y t}+u_{x x y}-3 u_{x x} u_{y}-3 u_{x} u_{x y}+\beta u_{x y}=0$

Where $\beta$ is a non-zero constant. According to the method by using Eq. (15), transform equation (36) into an ordinary differential equation, integrating ODE and neglecting constants of integration we have:

$k^{3} u^{\prime \prime \prime}+(k \beta-c) u^{\prime}-3 k^{2}\left(u^{\prime}\right)^{2}=0$

Where the prime denotes differential with respect to $\xi$. Balancing $u$ "' with $\left(u^{\prime}\right)^{2}$, we get $n+3=2(n+1)$ hence $n=1$. Hence for $n=1 \mathrm{Eq}$. (12) reduces to

$u(\xi)=\alpha_{0}+\alpha_{1}(m+F)+\beta_{1}(m+F)^{-1}$

Now substituting Eq. (38) along with Eq. (14) into Eq. (37), we get a polynomial in $F(\xi)$.Equating the coefficient of same power of $F(\xi)$, we attain a system of algebraic equations that consists of nine algebraic equations ( for simplicity, we omitted to display them). Solving these systems of equations for $\alpha_{0}, \alpha_{1}, \beta_{1}, m$ and $c$ with the aid of MAPLE 17, the following sets of solutions are obtained:

Set- 01:

$$
\begin{aligned}
& c=k^{3} \lambda^{2}-4 k^{3} \mu+k \beta, m=m, \alpha_{0}=\alpha_{0}, \alpha_{1}=0, \\
& \beta_{1}=2 k m^{2}-2 k m \lambda+2 k \mu
\end{aligned}
$$

Set- 02:

$c=k^{3} \lambda^{2}-4 k^{3} \mu+k \beta, m=m, \alpha_{0}=\alpha_{0}, \alpha_{1}=-2 k, \beta_{1}=0$

Set-3:

$$
\begin{aligned}
& c=4 k^{3} \lambda^{2}-16 k^{3} \mu+k \beta, m=\frac{1}{2} \lambda, \alpha_{0}=\alpha_{0}, \alpha_{1}=-2 k, \\
& \beta_{1}=-\frac{1}{2} k \lambda^{2}+2 k \mu
\end{aligned}
$$

\subsubsection{Hyperbolic function solutions}

When $\Omega=\lambda^{2}-4 \mu>0$ we obtain the hyperbolic function solutions of Eq. (36).

Family 1:

$u_{1}(\xi)=\alpha_{0}+\frac{2 k m^{2}-2 k m \lambda+2 k \mu}{m+\frac{1}{2} \sqrt{\Omega} \operatorname{coth}\left(A+\frac{1}{2} \sqrt{\Omega} \xi\right)-\frac{1}{2} \lambda}$

$u_{2}(\xi)=\alpha_{0}+\frac{2 k m^{2}-2 k m \lambda+2 k \mu}{m+\frac{1}{2} \sqrt{\Omega} \tanh \left(A+\frac{1}{2} \sqrt{\Omega} \xi\right)-\frac{1}{2} \lambda}$

Where $\xi=k x+r y-\left(k^{3} \lambda^{2}-4 k^{3} \mu+k \beta\right) t$

Family 2:

$u_{3}(\xi)=\alpha_{0}-2 k\left(m+\frac{1}{2} \sqrt{\Omega} \operatorname{coth}\left(A+\frac{1}{2} \sqrt{\Omega} \xi\right)-\frac{1}{2} \lambda\right)$

$u_{4}(\xi)=\alpha_{0}-2 k\left(m+\frac{1}{2} \sqrt{\Omega} \tanh \left(A+\frac{1}{2} \sqrt{\Omega} \xi\right)-\frac{1}{2} \lambda\right)$

Where $\xi=k x+r y-\left(k^{3} \lambda^{2}-4 k^{3} \mu+k \beta\right) t$

Family 3: 


$$
\begin{gathered}
u_{5}(\xi)=\alpha_{0}-k \sqrt{\Omega} \operatorname{coth}\left(A+\frac{1}{2} \sqrt{\Omega} \xi\right)+\frac{2\left(-\frac{1}{2} k \lambda^{2}+2 k \mu\right)}{\sqrt{\Omega} \operatorname{coth}\left(A+\frac{1}{2} \sqrt{\Omega} \xi\right)} \\
u_{6}(\xi)=\alpha_{0}-k \sqrt{\Omega} \operatorname{coth}\left(A+\frac{1}{2} \sqrt{\Omega} \xi\right) \\
+\frac{2\left(-\frac{1}{2} k \lambda^{2}+2 k \mu\right)}{\sqrt{\Omega} \operatorname{coth}\left(A+\frac{1}{2} \sqrt{\Omega} \xi\right)}
\end{gathered}
$$

Where $\xi=k x+r y-\left(4 k^{3} \lambda^{2}-16 k^{3} \mu+k \beta\right) t$

\subsubsection{Trigonometric function solutions}

When $\Omega=\lambda^{2}-4 \mu<0$, we obtain the trigonometric function solutions of Eq. (36).

Family 4:

$$
\begin{aligned}
& u_{7}(\xi)=\alpha_{0}+\frac{2 k m^{2}-2 k m \lambda+2 k \mu}{m+\frac{1}{2} \sqrt{-\Omega} \tan \left(A-\frac{1}{2} \sqrt{-\Omega} \xi\right)-\frac{1}{2} \lambda} \\
& u_{8}(\xi)=\alpha_{0}+\frac{2 k m^{2}-2 k m \lambda+2 k \mu}{m+\frac{1}{2} \sqrt{-\Omega} \tan \left(A-\frac{1}{2} \sqrt{-\Omega} \xi\right)-\frac{1}{2} \lambda}
\end{aligned}
$$

Where $\xi=k x+r y-\left(k^{3} \lambda^{2}-4 k^{3} \mu+k \beta\right) t$

Family 5:

$$
\begin{aligned}
& u_{9}(\xi)=\alpha_{0}-2 k\left(m+\frac{1}{2} \sqrt{-\Omega} \cot \left(A+\frac{1}{2} \sqrt{-\Omega} \xi\right)-\frac{1}{2} \lambda\right) \\
& u_{10}(\xi)=\alpha_{0}-2 k\left(m+\frac{1}{2} \sqrt{-\Omega} \tan \left(A-\frac{1}{2} \sqrt{-\Omega} \xi\right)-\frac{1}{2} \lambda\right)
\end{aligned}
$$

Where $\xi=k x+r y-\left(k^{3} \lambda^{2}-4 k^{3} \mu+k \beta\right) t$

Family 6:

$$
\begin{array}{r}
u_{11}(\xi)=\alpha_{0}-k \sqrt{-\Omega} \cot \left(A+\frac{1}{2} \sqrt{-\Omega} \xi\right) \\
+\frac{2\left(-\frac{1}{2} k \lambda^{2}+2 k \mu\right)}{\sqrt{-\Omega} \cot \left(A+\frac{1}{2} \sqrt{-\Omega} \xi\right)} \\
u_{12}(\xi)=\alpha_{0}-k \sqrt{-\Omega} \tan \left(A-\frac{1}{2} \sqrt{-\Omega} \xi\right) \\
+\frac{2\left(-\frac{1}{2} k \lambda^{2}+2 k \mu\right)}{\sqrt{-\Omega} \tan \left(A-\frac{1}{2} \sqrt{-\Omega} \xi\right)}
\end{array}
$$

$$
u_{13}(\xi)=\alpha_{0}+\frac{2 k m^{2}-2 k m \lambda+2 k \mu}{m+\frac{B}{B \xi+A}-\frac{1}{2} \lambda}
$$

Where $\xi=k x+r y-\left(k^{3} \lambda^{2}-4 k^{3} \mu+k \beta\right) t$

Family 8:

$u_{14}(\xi)=\alpha_{0}-2 k\left(m+\frac{B}{B \xi+A}-\frac{1}{2} \lambda\right)$

Where $\xi=k x+r y-\left(k^{3} \lambda^{2}-4 k^{3} \mu+k \beta\right) t$

Family 9:

$u_{15}(\xi)=\alpha_{0}-\frac{2 k B}{B \xi+A}+\frac{\left(-\frac{1}{2} k \lambda^{2}+2 k \mu\right)(B \xi+A)}{B}$

Where $\xi=k x+r y-\left(4 k^{3} \lambda^{2}-16 k^{3} \mu+k \beta\right) t$

\section{Results and discussion}

In this present work, we have solved extended shallow water wave equations and found fifteen solutions, but author [22] have found only six solutions by using basic $\left(G^{\prime} / G\right)$-expansion method. From our observation, we found that if we set $m=0$ and leave out the portion $\sum_{i=1}^{n} \beta_{i}(m+F(\xi))^{-i}$ in our solution, then our obtained solution coincides with Biker [22] solution. Hence, we conclude that the basic $\left(G^{\prime} / G\right)$-expansion method established by Wang et al. [20] is the particular case of the improved $\left(G^{\prime} / G\right)$-expansion method. Comparing our obtained results from equations (14) and (36), it can be seen that the results are same with Biker [22] solutions when we set $m=0$ and leave out the portion $\sum_{i=1}^{n} \beta_{i}(m+F(\xi))^{-i}$ in our solution. Similarly, for extended $(3+1)$ dimensional shallow water wave equations, using the wave variable $\xi=k x+r y+s z-c t$ equations (7) and (8) must be carried into the ordinary differential equations (16) and (37) respectively. Thus the improved $\left(G^{\prime} / G\right)$-expansion method is applied to these equations, it can be seen that the obtained solutions are the same with solutions of equations (16) and (37).From our obtained solutions we observe that solutions from family 1 to family 3 are hyperbolic function solutions for $\lambda^{2}-4 \mu>0$, from Family 4 to Family 6 are trigonometric function solutions for $\lambda^{2}-4 \mu<0$ and from Family 7 to Family 9 are rational function solutions for $\lambda^{2}-4 \mu=0$. When the parameters are taken as special values, the solitary wave solutions and periodic wave solutions are derived from the hyperbolic function solution and trigonometric function solution, respectively.

Where $\xi=k x+r y-\left(4 k^{3} \lambda^{2}-16 k^{3} \mu+k \beta\right) t$

\subsubsection{Rational function solutions}

When $\Omega=\lambda^{2}-4 \mu=0$, we obtain the following solutions of Eq.

(36).

Family 7: 


\section{Graphical representations}

In this section, we will discuss about the nature of some obtained solutions of the equation (14) and (36) by selecting particular values of the parameters existing in the exact solutions use the mathematical software Maple 17, which are represented in Fig. 1-9

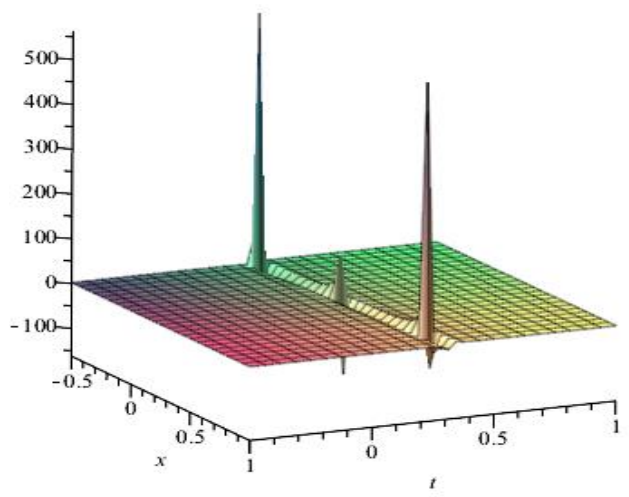

Fig. 1: 3D Graphics (Soliton Profile) of Solutions Eq. (21) when $\alpha_{0}=3, k=0.8, r=2, \beta=5, \lambda=3, \mu=1, m=1, y=0, A=5$ and $-.5 \leq x, t \leq 1$

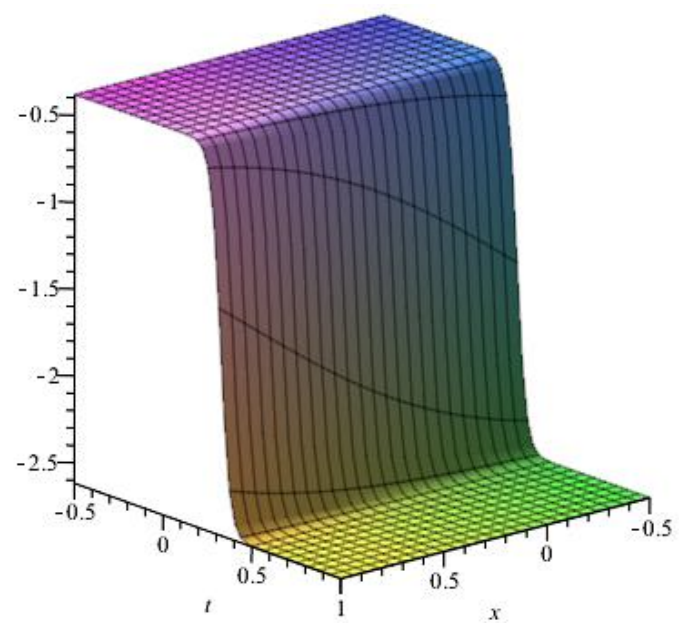

Fig. 2: 3D Graphics (Kink Profile) of Solutions Eq. (24) when $\alpha_{0}=3, k=0.8, r=2, \beta=5, \lambda=3, \mu=1, m=1, y=0, A=5$ and $-.5 \leq x, t \leq 1$.

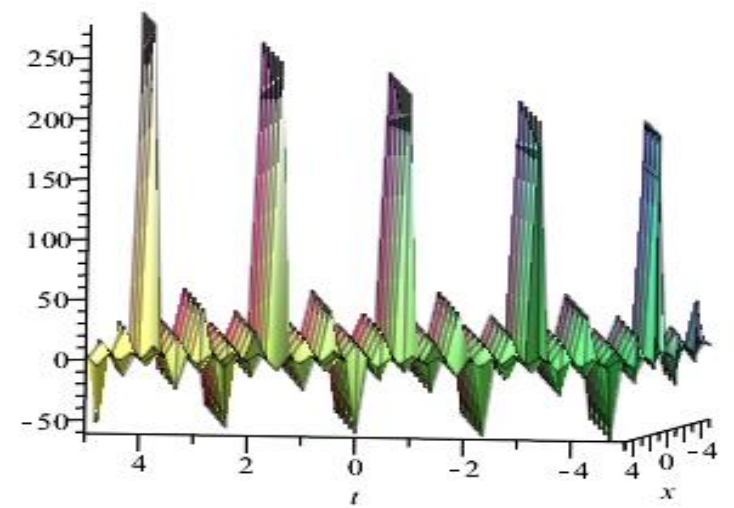

Fig. 3: 3D Graphics (Periodic Profile) of Solutions Eq. (27) when $\alpha_{0}=3, k=1, r=1, \beta=2, \lambda=1, \mu=2, m=0.5, y=0, A=5$ and $-5 \leq x, t \leq 5$.

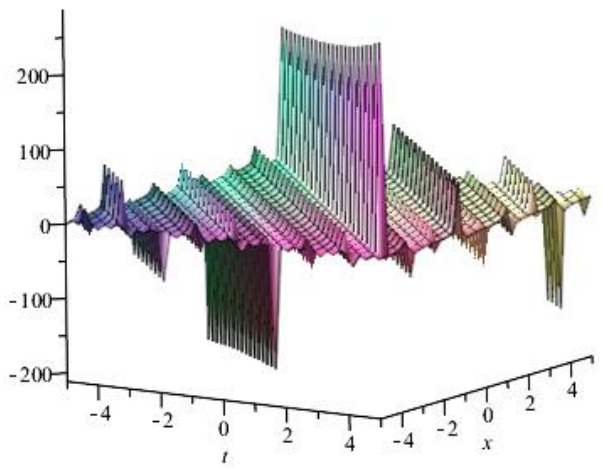

Fig. 4: 3D Graphics (Periodic Profile) of Solutions Eq. (49) when $\alpha_{0}=3, k=1, r=1, \beta=3, \lambda=1, \mu=2, m=0.5, y=0, A=5$ and $-5 \leq x, t \leq 5$.

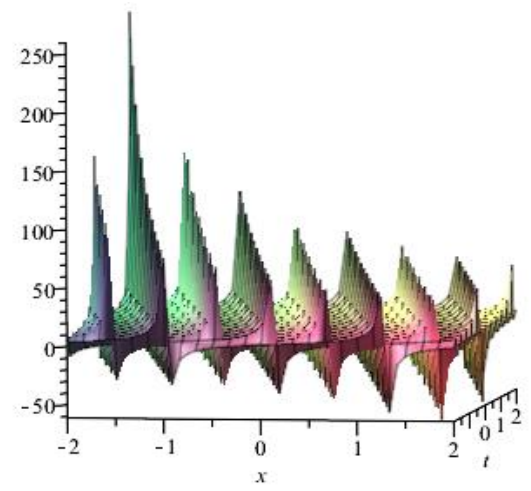

Fig. 5: 3D Graphics (Periodic Profile) of Solutions Eq. (28) when $\alpha_{0}=5, k=-8, r=-1, \beta=2, \lambda=.2, \mu=3, m=1, y, A=0$ and $-2 \leq x, t \leq 2$

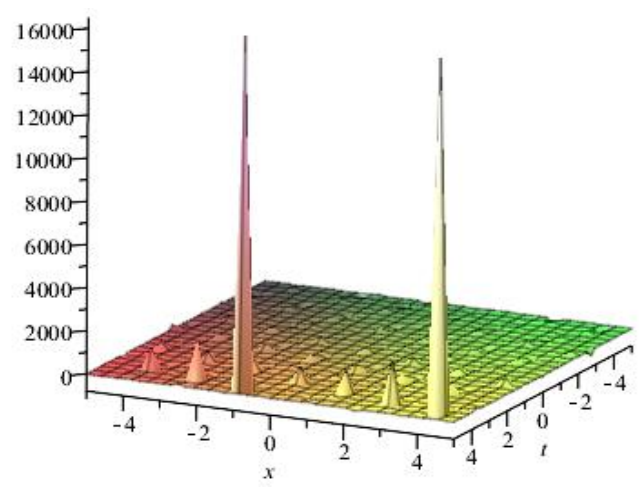

Fig. 6: 3D Graphics (Soliton Profile) of Solutions Eq. (53) when $\alpha_{0}=3, k=1, r=1, \beta=3, \lambda=1, \mu=2, m=0.5, y=0, A=5$ and $-5 \leq x, t \leq 5$.

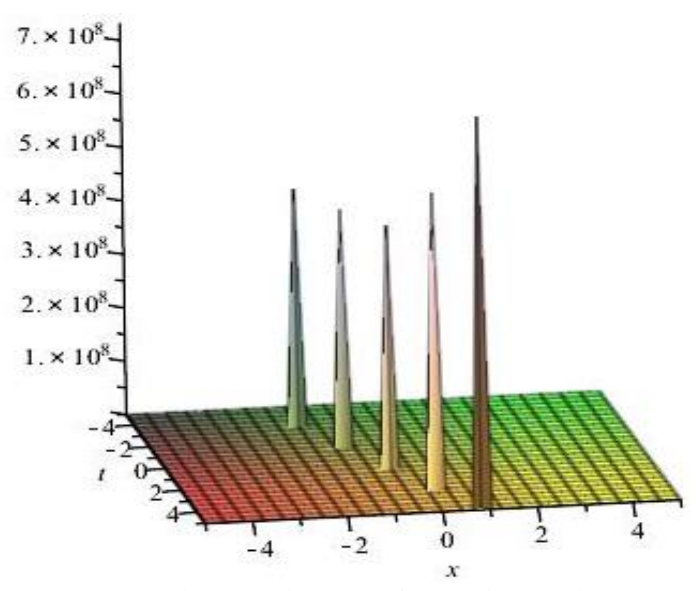

Fig. 7: $3 \mathrm{D}$ Graphics (Soliton Profile) of Solutions Eq. (55) when $\alpha_{0}=3, k=1.5, r=-0.5, \beta=3, \lambda=2, \mu=1, m=1, y=0, A=5, B=1 \mathrm{a}$ nd $-5 \leq x, t \leq 5$. 


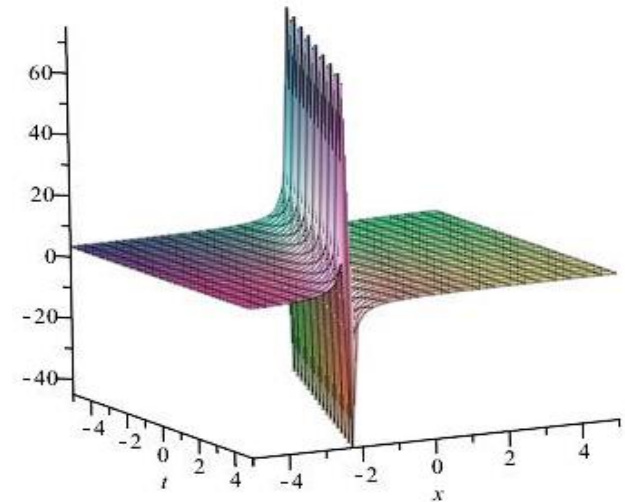

Fig. 8: $3 D$ Graphics (Singular Kink Profile) of Solutions Eq. (34) when $\alpha_{0}=3, k=1.5, r=-0.5, \beta=1, \lambda=2, \mu=1, m=1, y=0, A=1, B=1 \quad$ And $-5 \leq x, t \leq 5$.

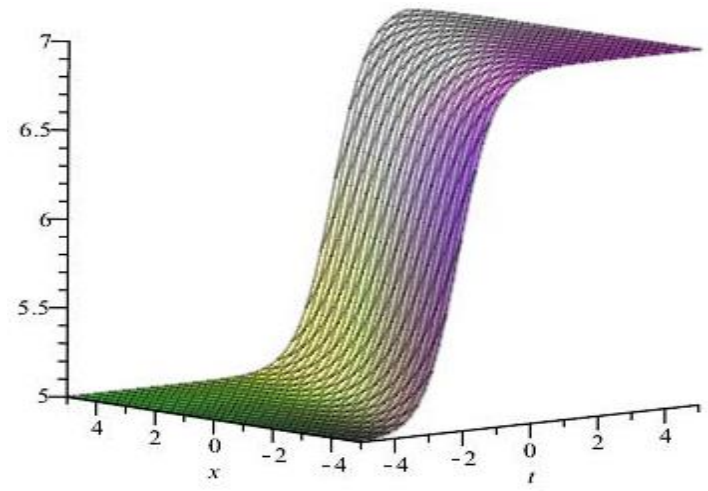

Fig. 9: 3D Graphics (Singular Kink Profile) of Solutions Eq. (56) when $\alpha_{0}=3, k=1.5, r=-0.5, \beta=3, \lambda=2, \mu=1, m=1, y=0, A=5, B=1 \quad$ and $-5 \leq x, t \leq 5$.

\section{Conclusion}

In this paper, we have examined the exact solutions for $(2+1)$ and $(3+1)$ dimensional extended shallow water wave equations involving parameters by applying the improved $\left(G^{\prime} / G\right)$-expansion method. Some of these results are in agreement with the results reported by others in the literature, and new results are formally developed in this work. The solution procedure is very simple and the travelling wave solutions are expressed in terms of hyperbolic functions, trigonometric functions and rational functions. It is shown that the improved $\left(G^{\prime} / G\right)$-expansion method provides a very effective and powerful mathematical tool for solving nonlinear equations in mathematical physics.

\section{References}

[1] Hirota, R. (1976). Direct method of finding exact solutions of nonlinear evolution equations. In Bäcklund transformations, the inverse scattering method, solitons, and their applications (pp. 40-68). Springer Berlin Heidelberg. https://doi.org/10.1007/BFb0081162.

[2] Wazwaz, A. M. (2005). The tanh method: solitons and periodic solutions for the Dodd-Bullough-Mikhailov and the Tzitzeica-DoddBullough equations. Chaos, Solitons \& Fractals, 25(1), 55-63. https://doi.org/10.1016/j.chaos.2004.09.122.

[3] Wazwaz, A. M. (2005). The tanh method: solitons and periodic solutions for the Dodd-Bullough-Mikhailov and the Tzitzeica-DoddBullough equations. Chaos, Solitons \& Fractals, 25(1), 55-63. https://doi.org/10.1016/j.chaos.2004.09.122.

[4] Kumar, D., \& Sarker, P. (2016). Investigation of exact traveling wave solution for the $(2+1)$ dimensional nonlinear evolution equations via modified extended tanh-function method. International Journal of Physical Research,4(2), 62-68. https://doi.org/10.14419/ijpr.v4i2.6588.

[5] Naher, H., Abdullah, F. A., \& Akbar, M. A. (2011). The expfunction method for new exact solutions of the nonlinear partial dif- ferential equations. International Journal of Physical Sciences, 6(29), 6706-6716. https://doi.org/10.5897/IJPS11.1026.

[6] Akbar, M. A., \& Ali, N. H. M. (2011). Exp-function method for Duffing Equation and new solutions of $(2+1)$ dimensional dispersive long wave equations. Progress in Applied Mathematics, 1(2), 30-42.

[7] Bekir, A., \& Aksoy, E. (2013). Exact solutions of extended shallow water wave equations by exp-function method. International Journal of Numerical Methods for Heat \& Fluid Flow, 23(2), 305-319. https://doi.org/10.1108/09615531311293489.

[8] Yusufoğlu, E., \& Bekir, A. (2006). Solitons and periodic solutions of coupled nonlinear evolution equations by using the sine-cosine method. International Journal of Computer Mathematics, 83(12), 915-924. https://doi.org/10.1080/00207160601138756.

[9] Vakhnenko, V. O., Parkes, E. J., \& Morrison, A. J. (2003). A Bäcklund transformation and the inverse scattering transform method for the generalised Vakhnenko equation. Chaos, Solitons \& Fractals, 17(4), 683-692. https://doi.org/10.1016/S09600779(02)00483-6.

[10] Dai, C., \& Zhang, J. (2006). Jacobian elliptic function method for nonlinear differential-difference equations. Chaos, Solitons \& Fractals, 27(4), 1042-1047. https://doi.org/10.1016/j.chaos.2005.04.071.

[11] Liu, S., Fu, Z., Liu, S., \& Zhao, Q. (2001). Jacobi elliptic function expansion method and periodic wave solutions of nonlinear wave equations. Physics Letters A, 289(1), 69-74. https://doi.org/10.1016/S0375-9601(01)00580-1.

[12] Mohyud-Din, S. T., \& Noor, M. A. (2009). Homotopy perturbation method for solving partial differential equations. Zeitschrift für Naturforschung A,64(3-4), 157-170. https://doi.org/10.1515/zna2009-3-402.

[13] Fan, E., \& Zhang, H. (1998). A note on the homogeneous balance method. Physics Letters A, 246(5), 403-406. https://doi.org/10.1016/S0375-9601(98)00547-7.

[14] Zhang, S., \& Xia, T. (2007). A generalized new auxiliary equation method and its applications to nonlinear partial differential equations. Physics Letters A, 363(5), 356-360. https://doi.org/10.1016/j.physleta.2006.11.035.

[15] Singh, S. S. (2016). Solutions of Kudryashov-Sinelshchikov equation and generalized Radhakrishnan-Kundu-Lakshmanan equation by the first integral method. International Journal of Physical Research, 4(2), 37-42. https://doi.org/10.14419/ijpr.v4i2.6202.

[16] Feng, Z., \& Wang, X. (2003). The first integral method to the twodimensional Burgers-Korteweg-de Vries equation. Physics Letters A, 308(2), 173-178. https://doi.org/10.1016/S0375-9601(03)000161 .

[17] Biazar, J., \& Aslanpanah, Z. (2012). The First Integral Method for the Generalized Drinfel'd-Sokolov-Wilson system and Bretherton equation. International Journal of Applied, 1(4), 634-642.

[18] Wazwaz, A. M. (2008). Multiple-soliton solutions of two extended model equations for shallow water waves. Applied Mathematics and Computation, 201(1), 790-799. https://doi.org/10.1016/j.amc.2008.01.017.

[19] Wazwaz, A. M. (2010). Multiple-soliton solutions for extended shallow water wave equations. Studies in Mathematical Sciences, 1(1), 21-29.

[20] Wang, M., Li, X., \& Zhang, J. (2008). The (G'/G)-expansion method and travelling wave solutions of nonlinear evolution equations in mathematical physics. Physics Letters A,372(4), 417-423. https://doi.org/10.1016/j.physleta.2007.07.051.

[21] Verma, A., Jiwari, R., \& Kumar, J. (2013). Traveling Wave Solutions for Shallow Water Wave Equation by $\left(\mathrm{G}^{\prime} / \mathrm{G}\right)$-Expansion Method. World Academy of Science, Engineering and Technology, International Journal of Mathematical, Computational, Physical, Electrical and Computer Engineering, 7(5), 817-821.

[22] Bekir, A., \& Aksoy, E. (2012). Exact solutions of shallow water wave equations by using the $(G \% G)$-expansion method. Waves in Random and Complex Media,22(3), 317-331. https://doi.org/10.1080/17455030.2012.683890.

[23] Islam, M. S., Khan, K., \& Akbar, M. A. (2015). An analytical method for finding exact solutions of modified Korteweg-de Vries equation. Results in Physics, 5, 131-135. https://doi.org/10.1016/j.rinp.2015.01.007.

[24] Khan, K., \& Akbar, M. A. (2014). Traveling wave solutions of nonlinear evolution equations via the enhanced $\left(\mathrm{G}^{\prime} / \mathrm{G}\right)$-expansion method. Journal of the Egyptian Mathematical Society, 22(2), 220226. https://doi.org/10.1016/j.joems.2013.07.009. 